\title{
A Photonic-Assisted Generation of Triangular Waveform With Frequency Octupling
}

\author{
Jin Yuan ( $\nabla$ yuanjin@cuc.edu.cn ) \\ Communication University of China \\ Jianxun Su \\ Communication University of China \\ Zengrui Li \\ Communication University of China
}

\section{Research Article}

Keywords: Triangular pulse, Frequency octupling, Modulation, SMF

Posted Date: January 4th, 2021

DOI: https://doi.org/10.21203/rs.3.rs-133788/v1

License: (c) (1) This work is licensed under a Creative Commons Attribution 4.0 International License. Read Full License 


\title{
A Photonic-assisted Generation of Triangular Waveform With Frequency Octupling
}

\author{
Jin Yuan*, Jianxun Su, Zengrui Li \\ State Key Laboratory of Media Convergence and Communication. \\ Communication University of China, Beijing, 100044, China
}

\begin{abstract}
A new photonic approach for generating a triangular waveform with octupled-frequency is presented. The core principle is the frequency outupling technique based on two cascaded dual-parallel Mach-Zehnder modulators (DP-MZMs). A dual-electrode MZM (De-MZM) and a single mode fiber (SMF) are subsequently applied to manipulate the signal spectrum to satisfy the characteristics of that of a triangular waveform. By applying a 2-GHz radio frequency (RF) signal, a full-duty-cycle triangular waveform with repetition rate of $16-\mathrm{GHz}$ is obtained. The high frequency multiplying factor shows great potential in generating a cost-effective waveform. Additionally, the phase imbalance of hybrid coupler and bias drift of MZM have been considered in our simulation, which further verify the feasibility and stability of our proposal.
\end{abstract}

Keywords: Triangular pulse, Frequency octupling, Modulation, SMF.

Corresponding Author: Jin Yuan Tel: $18301609662 \quad$ E-mail: yuanjin@cuc.edu.cn

\section{INTRODUCTION}

Recently, photonic techniques have been used in microwave waveform generation and manipulation thanks to the superiority of high frequency, large bandwidth and anti-electromagnetic interference (EMI) compared to the traditional electronic techniques $[1,2]$. Among microwave waveforms, a triangular waveform featured with linear rising /falling edge in intensity has attracted much attention due to its wide applications in all-optical network, such as signal copying, signal compression, wavelength conversion and so on [3-5].

Many photonic-assisted methods using different implementation principles for triangular waveform generation have been reported. One of the popular triangular waveform generation is based on the frequency-to-time mapping (FTTM) technique [6-7]. As in Ref. [6], an all-fiber scheme based on FTTM using two filter modules to generate a triangular waveform has been reported. In Ref. [7], a photonic arbitrary waveform generation using crossed FTTM combined with intersymbol interference is performed. The inadequacies of such schemes are the high cost and low flexibility. The duty cycle of triangular waveform generated by this kind of scheme is generally less than 1. Another common method of triangular waveform generation is using an optoelectronic oscillator (OEO) [8-11]. In Ref. [9], a full-duty-cycle triangular waveform is generated using an OEO loop in which a polarization modulator (PolM) is involved. In Ref. [10], by establishing a dual-loop OEO configuration and properly controlling the modulation mode in a Mach-Zehnder modulator (MZM), a high-quality 
triangular waveform signal is achieved. In Ref. [11], an OEO which can concurrently oscillate at two orthogonally polarized frequencies is applied to generate a triangular pulse train. In these schemes, no additional radio frequency (RF) source is required. In addition, triangular waveform generation based on time-domain synthesis (TDS) has also been proposed recently [12-16]. In Ref. [12, 13], Y. Jiang et al. proposed photonic approaches to generate microwave waveforms by synthesizing and carving optical field envelopes in time-domain. In Ref. [14-16], triangular waveform generation methods based on waveform time-domain synthesis in MZMs at different modulation modes are presented. In addition to the proposals mentioned above, schemes based on external modulation cooperated with spectrum manipulation are also widely studied [17-24]. A continuous wave (CW) is firstly modulated by an external modulator, after which a sequence of optical spectral lines is generated. Then various photonic techniques, like dispersion elements [17, 18], filters [19-21], or optical nonlinear effect [22-24] are applied to manipulate the amplitudes and phases of the spectral lines to satisfy the characteristics of a triangular waveform. For example, as in Ref. [17], J. Li et al. proposed a triangular pulse generator based on a dual-electrode MZM (De-MZM) and a normal dispersion fiber (NDF). In Ref. [19], a notch filter is applied to manipulate the spectrum of the phase-modulated signal. In Ref. [24], phase shift induced by the gain-transparent stimulated Brillouin scattering (SBS) act as a spectrum manipulation technique in the triangular waveform generation. The triangular waveform generated by the scheme based on external modulation has a full duty cycle. However, the repetition rate of the obtained periodical triangular waveform is generally equal to or double of the applied RF sinusoidal signal. Frequency multiplying can realize the generation of signals with high repetition rate by using lower-frequency RF signals, which enhances the effectivity and reduces the cost. Therefore, generating a stable triangular waveform with high frequency multiplying factor is an important issue.

In the previous research, generation of triangular waveform with frequency doubling [20] and frequency quadrupling [25] have been proposed. In this work, we propose a novel photonic approach to generate a full-duty-cycle triangular waveform with frequency octupling. The generator is composed of three parts: frequency octupling structure, De-MZM, and a piece of single mode fiber (SMF). Two cascaded dual-parallel MZMs (DP-MZMs) are used to implement the frequency octupling, after which the frequency interval of the modulated sidebands is octupling of the applied RF signal and a frequency-octupling electrical signal is obtain after detection in a PIN. Subsequently, optical double sideband (ODSB) modulation in a De-MZM and phase shift of a SMF is utilized to control the spectrum aligning with that of a triangular waveform. Based on this scheme, a 16-GHz triangular waveform is achieved by using a 2-GHz RF sinusoidal signal

\section{RESULTS}

\section{Design of triangular-shaped waveform generator.}




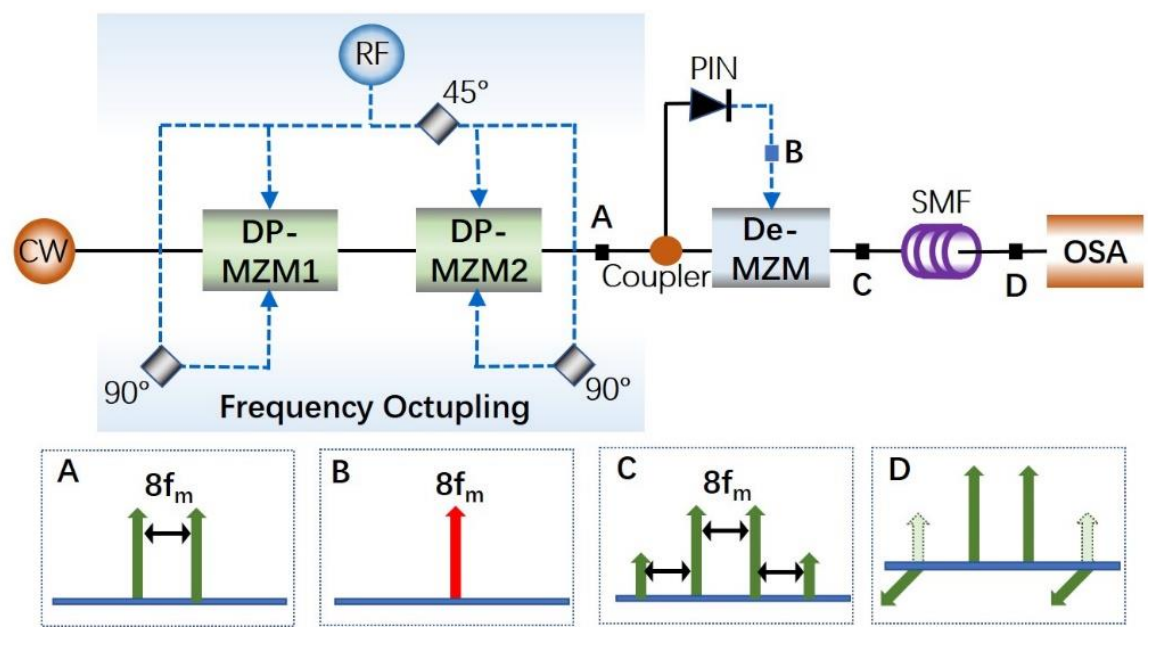

Fig. 1. Schematic diagram of the proposed triangular waveform generator.

The proposed frequency-octupled triangular waveform generator is as shown in Fig.1. The optical field of the input CW light is defined as $E_{i n}(t)=E_{0} \cos \left(\omega_{0} t\right)$, where $E_{0}$ and $\omega_{0}$ represent the amplitude and frequency, respectively. The applied electrical RF driving signal from a local oscillator (LO) is $V_{m}(t)=V_{m} \cos \left(\omega_{m} t\right)$. The frequency octupling structure consists of two cascaded DP-MZMs. For each DP-MZM, the two sub-MZMs are bias at the maximum transmission point (MATP), the main-MZM is biased at minimum transmission point (MITP), and a $90^{\circ}$ phase shift is introduced between the two RF driving signals of the sub-MZMs. A $45^{\circ}$ phase delay is introduced between the driving signals of DP-MZM1 and DP-MZM2. The driving signals of the sub-MZMs of DP-MZM2 can be expressed as $V_{m} \cos \left(\omega_{m} t+\pi / 4\right)$ and $V_{m} \cos \left(\omega_{m} t+3 \pi / 4\right)$, respectively.

\section{Frequency octupling technique}

The optical field of the signal at DP-MZM1 output can be expressed as [26]

$$
E_{D P-M Z M 1}(t)=-E_{0} \propto \sum_{n=1}^{\infty} J_{4 n-2}\left(m_{1}\right)\left(\begin{array}{l}
\cos \left\{\left[\omega_{0}+(4 n-2) \omega_{m}\right] t\right\} \\
+\cos \left\{\left[\omega_{0}-(4 n-2) \omega_{m}\right] t\right\}
\end{array}\right)
$$

wherein $J_{4 n-2}(m)$ denotes the Bessel function of the first kind of order $4 n-2$, parameterized by the modulation index of DP-MZM. The modulation index of DP-MZM is $m_{l}=\pi V_{m} / 2 V_{\pi l}$, where $V_{\pi l}$ is the half-wave switching voltage of DP-MZM1. As stated in our previous work [21], for a small signal modulation, harmonics higher than $J_{2}(m)$ can be ignored. Thus Eq. (1) can be simplified as

$$
E_{D P-M D M 1}(t)=-E_{0}\left\{J_{2}\left(m_{1}\right) \cos \left[\left(\omega_{0}+2 \omega_{m}\right) t\right]+J_{2}\left(m_{1}\right) \cos \left[\left(\omega_{0}-2 \omega_{m}\right) t\right]\right\}
$$

After remodulation in DP-MZM2, the output optical field at Point A can be calculated as 


$$
\begin{aligned}
E_{A}(t)= & \frac{1}{2} \cdot\left[-E_{0} J_{2}\left(m_{1}\right)\right] \cdot\left\{\cos \left[m_{2} \cdot \cos \left(\omega_{m} t+\frac{\pi}{4}\right)\right]-\cos \left[m_{2} \cdot \cos \left(\omega_{m} t+\frac{3 \pi}{4}\right)\right]\right\} \\
& =\frac{1}{2} \cdot\left[-E_{0} J_{2}\left(m_{1}\right)\right] \cdot\left\{\begin{array}{l}
J_{0}\left(m_{2}\right)+2 \sum_{n=1}^{\infty} J_{2 n}\left(m_{2}\right) \cdot(-1)^{n} \cos \left[n \cdot\left(2 \omega_{m} t+\frac{\pi}{2}\right)\right] \\
-J_{0}\left(m_{2}\right)-2 \sum_{n=1}^{\infty} J_{2 n}\left(m_{2}\right) \cdot(-1)^{n} \cos \left[n \cdot\left(2 \omega_{m} t+\frac{3 \pi}{2}\right)\right]
\end{array}\right\}
\end{aligned}
$$

The modulation index of DP-MZM2 is $m_{2}=\pi V_{m} / 2 V_{\pi 2}$. Similarly, ignoring the high harmonic components, Eq. (3) can be further simplified as

$$
E_{A}(t)=E_{0} J_{2}\left(m_{1}\right) J_{2}\left(m_{2}\right)\left\{-\sin \left[\left(\omega_{0}+4 \omega_{m}\right) t\right]+\sin \left[\left(\omega_{0}-4 \omega_{m}\right) t\right]\right\}
$$

Notably, only the fourth-order optical sidebands exist in the signal spectrum at the output of DP-MZM2, as shown visually in Figure 1(A). This optical signal is then coupled to a PIN for square-law detection, after which an electrical signal with a frequency octupling of the applied RF signal is acquired. Assuming that the detection responsibility is $\mathfrak{R}$, the corresponding photocurrent can be written as

$$
I_{B}(t) \propto \frac{1}{2} \mathfrak{R} J_{2}^{2}\left(m_{1}\right) J_{2}^{2}\left(m_{2}\right) \sin \left(8 \omega_{m} t\right)
$$

\section{Optical double sideband modulation}

This electrical signal at frequency of $8 \omega_{m}$ is used to drive the following De-MZM. A $90^{\circ}$ phase difference is introduced between the two driving signals of the dule electrode ports of De-MZM. When the bias point is at MATP, De-MZM is working at ODSB modulation state. The modulation index $m_{3}=\pi V_{m} / 2 V_{\pi 3}, V_{\pi 3}$ denotes the half-wave voltage of De-MZM. In the case of a small signal modulation, the optical field of signal at De-MZM output can be expressed as

$$
E_{C}(t) \propto\left\{\begin{array}{l}
J_{-1}\left(m_{3}\right) \exp \left(-j 12 \omega_{m} t\right)+\left[J_{0}\left(m_{3}\right)+J_{-1}\left(m_{3}\right)\right] \exp \left(-j 4 \omega_{m} t\right) \\
+\left[J_{0}\left(m_{3}\right)+J_{1}\left(m_{3}\right)\right] \exp \left(j 4 \omega_{m} t\right)+\left[J_{1}\left(m_{3}\right)\right] \exp \left[j 12 \omega_{m} t\right]
\end{array}\right\}
$$

Substituting $K_{4}=J_{0}\left(m_{3}\right)+J_{1}\left(m_{3}\right), K_{-4}=J_{0}\left(m_{3}\right)-J_{1}\left(m_{3}\right), K_{12}=J_{1}\left(m_{3}\right)$ and $K_{-12}={ }_{-} J_{1}\left(m_{3}\right)$ for simplification, the intensity can be calculated as

$$
I_{B}(t) \propto\left\{\begin{array}{l}
{\left[K_{-12}^{2}+K_{-4}^{2}+K_{4}^{2}+K_{12}^{2}\right]+2\left(K_{-4} K_{4}+K_{-12} K_{-4}+K_{-4} K_{-12}\right) \exp \left(j 8 \omega_{m} t\right)} \\
+2\left(K_{-12} K_{4}+K_{-4} K_{12}\right) \exp \left(j 16 \omega_{m} t\right)+2 K_{-12} K_{12} \exp \left(j 24 \omega_{m} t\right)
\end{array}\right\}
$$

In Eq.(7), component $\cos \left(16 \omega_{m} t\right)$ is undesired, and an SMF is inserted to remove its contribution. According to the SMF transmission function stated in Ref. [27], the phase of optical frequency item passing through a SMF is $\phi=2 \beta_{2} L \omega_{m}^{2}$, wherein $\beta_{2} L$ represents the dispersion. The optical field and intensity of signal after SMF can be calculated as follows. 


$$
\begin{gathered}
E_{D}(t) \propto\left\{\begin{array}{l}
K_{-12} \exp \left(-j 12 \omega_{m} t+j 18 \varphi\right)+K_{-4} \exp \left(-j 4 \omega_{m} t+j 2 \varphi\right) \\
+K_{4} \exp \left(j 4 \omega_{m} t+j 2 \varphi\right)+K_{12} \exp \left(j 12 \omega_{m} t+j 18 \varphi\right)
\end{array}\right\} \\
I_{C}(t) \propto\left\{\begin{array}{l}
{\left[K_{-12}^{2}+K_{-4}^{2}+K_{4}^{2}+K_{12}^{2}\right]+2 K_{-4} K_{4} \cos \left(8 \omega_{m} t\right)+2 K_{-12} K_{12} \cos \left(24 \omega_{m} t\right)} \\
+2 K_{12} K_{4} \cos (16 \varphi) \cos \left(8 \omega_{m} t\right)+2 K_{-6} K_{2} \cos (16 \varphi) \cos \left(16 \omega_{m} t\right)
\end{array}\right\}
\end{gathered}
$$

To remove the item of $\cos \left(16 \omega_{m} t\right)$, the SMF-induced phase shift $\phi=16 \varphi$ should be $\phi=(2 k+1) \pi / 2$. The required dispersion of SMF can be calculated as

$$
\beta_{2} L=\frac{(2 \mathrm{k}+1) \pi}{64 \omega_{m}^{2}}, k=0,1,2, \ldots
$$

For $k=0, \beta_{2} L$ is equal to $\pi / 64 \omega_{m}^{2}$. The relationship between $\beta_{2} L$ and the frequency of RF signal $f_{m}$ has been displayed in Fig.2 (a). We can eliminate the influence of $\cos \left(16 \omega_{m} t\right)$ by using an SMF with required dispersion value. The intensity of signal after $\mathrm{SMF}$ can be expressed as

$$
I_{D}(t) \propto I_{D C}+\cos \left(8 \omega_{m} t\right)+\frac{J_{1}^{2}\left(m_{3}\right)}{J_{1}^{2}\left(m_{3}\right)-J_{0}^{2}\left(m_{3}\right)} \cos \left(24 \omega_{m} t\right)
$$

The Fourier series expansion of a full-duty-cycle triangular waveform is described by

$$
T_{t r i}(t)=D+\sum_{n=1,3,5 \ldots}^{\infty} \frac{1}{n} \cos (n \Omega t) \propto \cos (\Omega t)+\frac{1}{9} \cos (3 \Omega t)+\frac{1}{25} \cos (5 \Omega t)+\mathrm{L}
$$

By comparing Eq. (11) and (12), the temporal intensity of the obtained waveform is consistent with the first two orders of the Fourier series expansion of $T_{t r}(t)$. To further meet the characteristics of a triangular waveform, the power difference $\Delta P$ between the first two items (8-th and 24-th harmonics in our case) should conform to the following formula.

$$
\Delta P=10 \log _{10}\left[\frac{J_{1}^{2}\left(m_{3}\right)-J_{0}^{2}\left(m_{3}\right)}{J_{1}^{2}\left(m_{3}\right)}\right]=9.5 \mathrm{~dB}
$$

The formula (13) is illustrated in Fig.2(b), which shown that to ensure $\Delta P$ is $9.5 \mathrm{~dB}$, the modulation index $m_{3}$ of De-MZM should be set as 0.6 . 

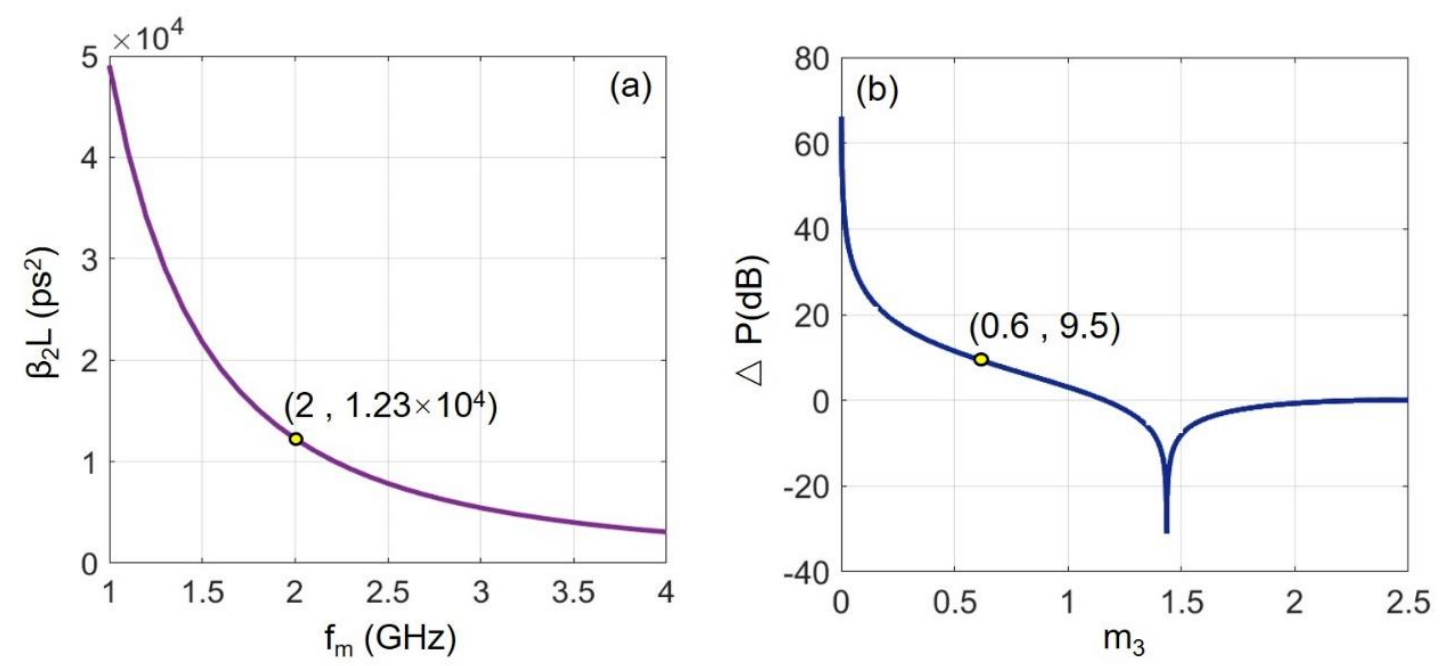

Fig. 2. (a) Values of $\beta_{2} L$ versus RF driving frequency, $f_{m}$. (b) Values of $\Delta P(\mathrm{~dB})$ versus modulation index of De-MZM, $m_{3}$.

\section{METHODS}

\section{Generation of Triangular-shaped waveform}

The feasibility of the proposed triangular waveform generator is verified in Optisystem. The schematic setup is as shown in Fig. 1. According to the theoretical analysis in Section 2, the parameters of the CW laser, DP-MZMs, De-MZM and SMF are listed in Table 1. A 2-GHz RF signal is applied as the driving frequency of DP-MZMs. By adjusting the amplitude of RF signal, both modulation indices of DP-MZMs are set as $m_{1}=m_{2}=1.2$. The modulation index of De-MZM is set as $m_{3}=$ 0.6. The following content gives the simulation results during the signal processing in triangular waveform generation.

Table.1 Simulation parameters of key devices

\begin{tabular}{c|lc}
\hline Device & \multicolumn{1}{c}{ Parameters } \\
\hline Laser & $\begin{array}{l}\text { Linewidth: } 1 \mathrm{MHz} \\
\text { Central Wavelength: } 1550 \mathrm{~nm}\end{array}$ & Output Power: $10 \mathrm{dBm}$ \\
\hline$M Z M$ & $\begin{array}{l}\text { Half-wave voltage: } 4 \mathrm{~V} \\
\text { Extinction ratio: } 30 \mathrm{~dB}\end{array}$ & Insertion loss: $5.5 \mathrm{~dB}$ \\
\hline$S M F$ & $\begin{array}{l}\text { Length: } 1.5 \mathrm{~km} \\
\text { Dispersion: } 1.23 \times 104 \mathrm{ps}^{2}\end{array}$ & Attenuation: $0.2 \mathrm{~dB} / \mathrm{km}$ \\
\hline
\end{tabular}

The optical spectrum of the output signal after DP-MZM2 is as shown in Fig.3 (a). Notably, through frequency octupling, two dominant optical sidebands are existed in the optical spectrum. Using a 2-GHz driving signal, the frequency spacing between the two dominant frequency items is $16 \mathrm{GHz}$. The undesired frequency items are suppressed more than $30 \mathrm{~dB}$ lower than the dominant sidebands. This frequency-octupled optical signal is then splitted into two paths, one of which is 
detected by a PIN and then used to drive the De-MZM, another path of optical signal is transmitted to the De-MZM for ODSB modulation. The electrical signal after photo-electric transform is as shown in Fig.3 (b). A pure electrical signal at $16 \mathrm{GHz}$ is obtained.
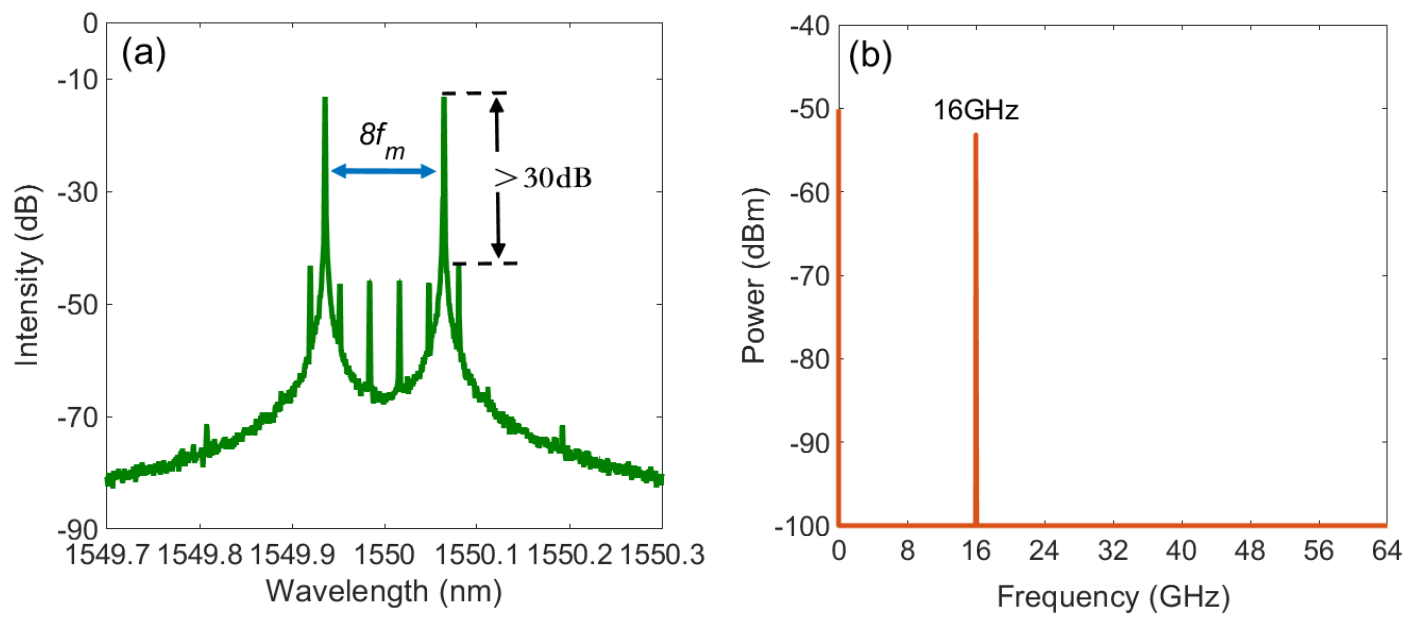

Fig. 3. Signal spectra after frequency octupling (a) optical spectrum at Point A, (b) Electrical spectrum at Point B.

As given in Fig. 4(a), after ODSB modulation in De-MZM, optical spectrum contains four main optical sidebands with an $8 f_{m}$ frequency interval. Figure 4 (b) shows the corresponding electrical spectrum, which contains a DC component and three frequency components of $16 \mathrm{GHz}, 24 \mathrm{GHz}$, and $48 \mathrm{GHz}$. This is in accordance with the expression of $I_{C}(t)$ in Eq. (9). There exist an undesired 32-GHz frequency component. As stated in Section 2, when the modulation index is $m_{3}=0.6$, the amplitude difference of the sidebands (4-th and 12-th) is $9.5 \mathrm{~dB}$, and the corresponding power difference $\left(\mathrm{P}_{16 \mathrm{GHz}} / \mathrm{P}_{48 \mathrm{GHz}}\right)$ is $\triangle P=19 \mathrm{~dB}$. The simulation results agree well with the theoretical results.
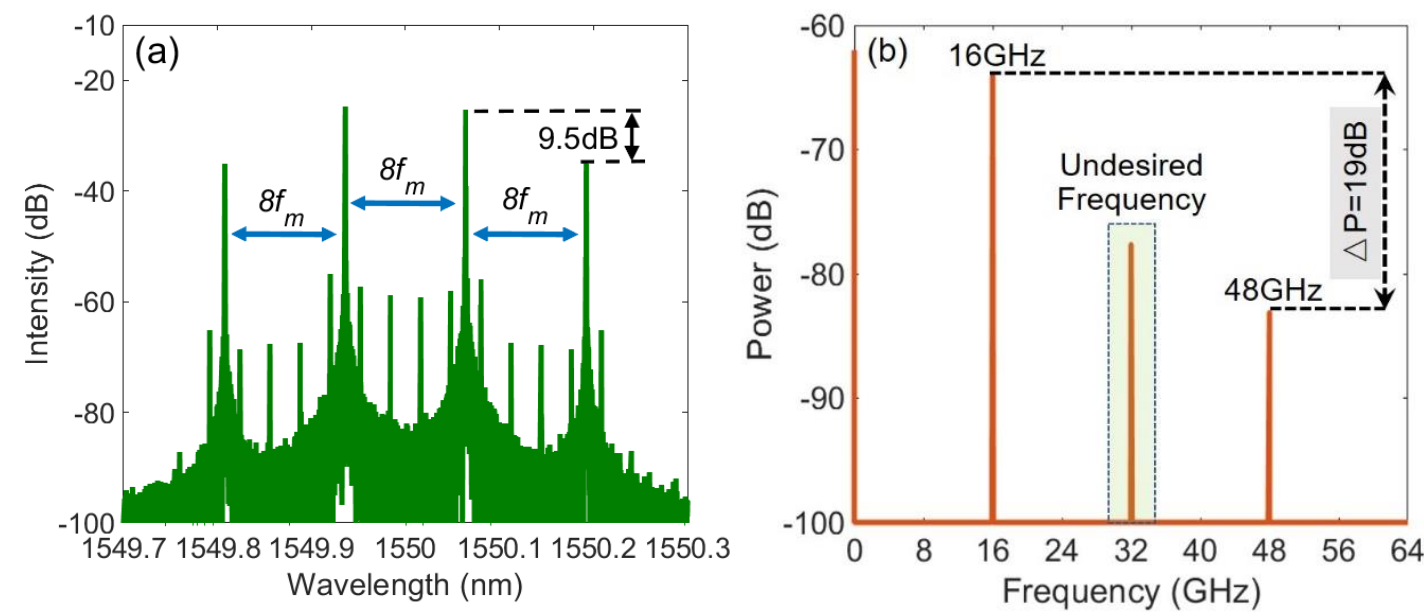

Fig. 4. Simulation results at output of De-MZM: (a) optical spectrum, (b) corresponding spectrum.

In the following simulation, we use an SMF to remove the undesired $32-\mathrm{GHz}$ frequency item. As in Fig.2(a), when the applied RF signal frequency is $2 \mathrm{GHz}$, the 
required fiber dispersion is $1.23 \times 104 \mathrm{ps}^{2}$. The output electrical spectrum is as shown in Fig. 5(a). The 32-GHz interference frequency component has been well suppressed, and the power difference of $\triangle P$ is still $19 \mathrm{~dB}$. The intensity distribution of the obtained signal is shown in Fig. 5(b). It shows that the acquired waveform is featured with a triangular shape, and the repetition rate is $16 \mathrm{GHz}$, octupling of the $2-\mathrm{GHz} \mathrm{RF}$ driving signal.
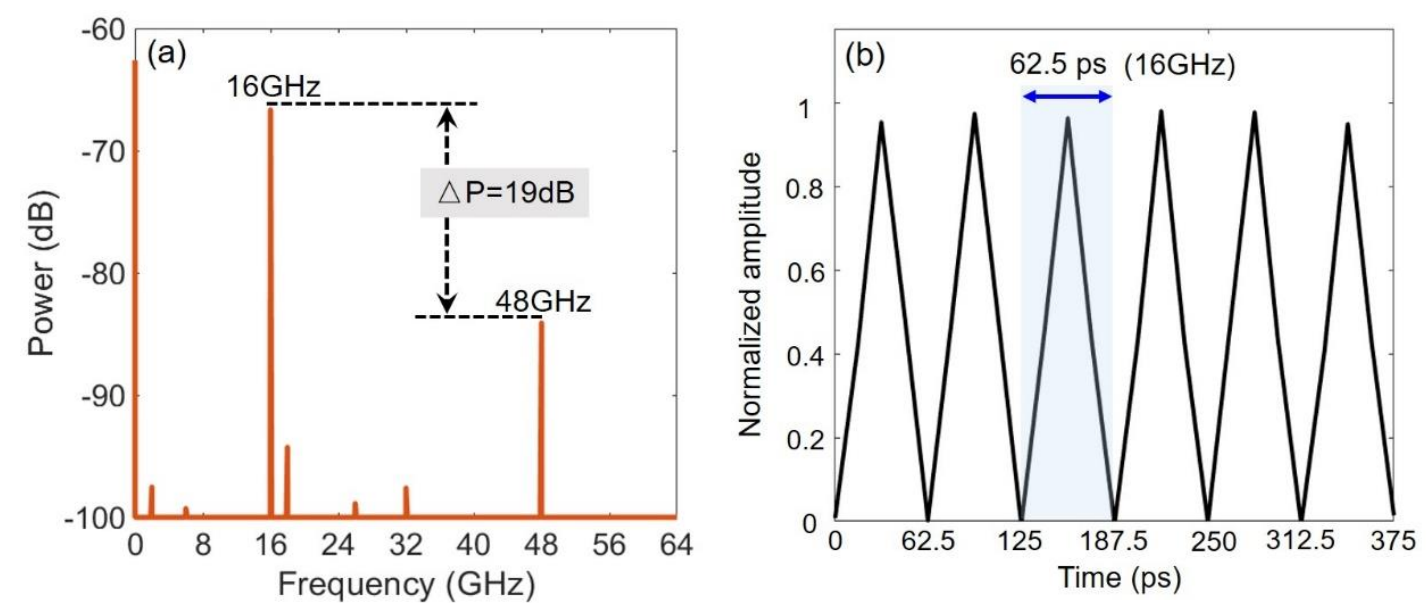

Fig. 5. (a) Optical spectrum, (b) the corresponding temporal waveform of the generated $16-\mathrm{GHz}$ triangular waveform.

\section{Analysis of hybrid coupler phase imbalance.}

In the frequency octupling processing, there exist a $45^{\circ}$ phase difference between the RF driving signals of the two cascaded DP-MZMs. Practically, the imbalance of the $45^{\circ}$ hybrid coupler may influence the characteristics of signals, which further affect the shape of the obtained triangular waveform. In Fig.6, we analyze the influence of a phase drift of $\pm 8 \%$ on the generated waveform. Figure 6(a) illustrates $\triangle P$ $\left(P_{16 \mathrm{GHz}} / P_{48 \mathrm{GHz}}\right)$ versus phase imbalance. It shows that the variation of $\triangle P$ is under $0.2 \mathrm{~dB}$ when the phase drift of $45^{\circ}$ hybrid coupler is under $\pm 8 \%$, which means that its influence on the two dominant frequency items is negligibly small. However, the phase imbalance may induce the undesired frequencies. Here, we introduce a concept of harmonic distortion suppression ratio (HDSR), denoting the power ratio between the 8 -th harmonic (16GHz in our case) and the maximum interference component. The relationship of HDSR versus the phase imbalance is calculated in Fig.6 (b). When the phase drift is within $\pm 2 \%, H D S R$ is larger than $22 \mathrm{~dB}$, which can be regard as an tolerable range. In Fig.6(c-d), we take different phase drifts for examples to analyze the generated temporal waveforms. It shows that the phase drift can influence the stability of peak values of periodic triangular waveform. For a $1.2 \%$ phase drift, the peak oscillation is $3.4 \%$. When the phase drift is beyond $2 \%$ ( $2.8 \%$ for example), the peak oscillation has a relatively high value of $16.7 \%$. We can approximately think that $\pm 2 \%$ is an acceptable range for the phase imbalance of the $45^{\circ}$ hybrid coupler. 

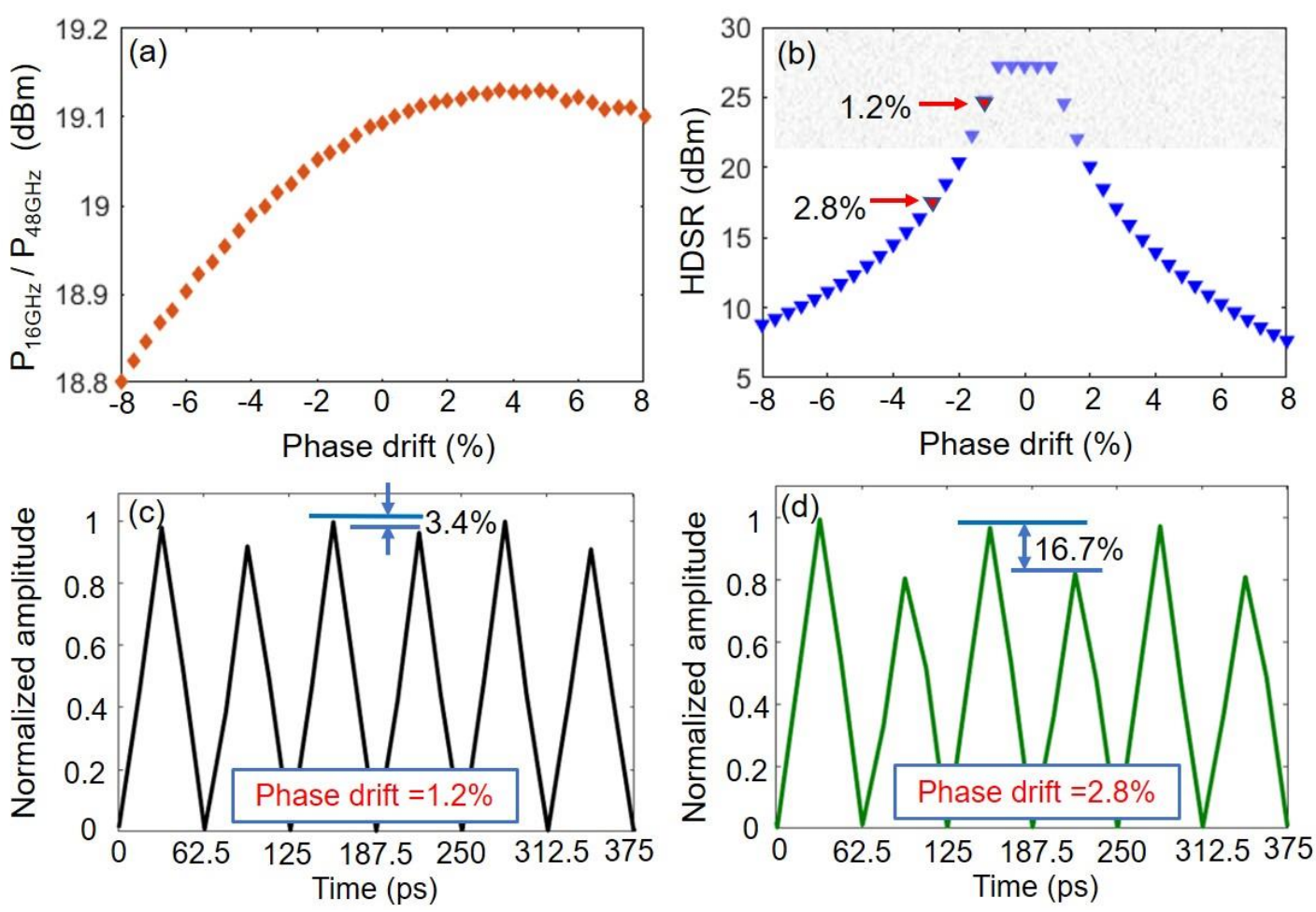

Fig. 6. (a-b) $\triangle P$ and $H D S R$ versus phase drift within a range of $\pm 8 \%$. (c-d) Temporal waveforms of the generated signal when phase drifts are $1.2 \%$ and $2.8 \%$.

\section{Analysis of bias point drift of DP-MZM.}

In addition, as key devices of the generation system, the drift of the MZM bias points should also be taken into account. In Fig.7, we take the bias drift of DP-MZM1 ranging from $-10 \%$ to $10 \%$ as an example for discussion. As can be seen from Fig.7(a), when the bias point drifting from $10 \%$ to $10 \%, \triangle P\left(P_{16 \mathrm{GHz}} / P_{48 \mathrm{GHz}}\right)$ is changing no more than $0.5 \%$ around $19 \mathrm{~dB}$. Indicating that the bias point drift has little effect on the dominant frequency components (DC component, 16-GHz and $18-\mathrm{GHz}$ components). Figure 7. (b) calculates the influence of the same bias drifting on the HDSR. Figure 7. (c-e) depict the signal electrical spectra with bias drifts of $8.5 \%, 5.5 \%$ and $3 \%$. With different bias drifts, three dominant frequency components are almost invariant while the interference components are induced. With a larger bias drift, the lower frequency region of the spectrum is more cluttered. Results in Fig. 7(f-h) are the corresponding temporal triangular waveforms. It can be seen that the undesired bias drift can weaken the slope linearity and interfere the peak stability of the generated triangular waveform. When the bias drifts are $8.5 \%, 5.5 \%$, and $3 \%$, the peak oscillation of temporal waveform are $19.6 \%, 11.3 \%$, and $5 \%$, respectively. Supposing that a vibration less than $5 \%$ is acceptable, the general tolerable range of bias drift is from $-3 \%$ to $3 \%$. The discussion of bias drifts of DP-MZM2 and De-MZM is similar work and we do not discuss them here. 

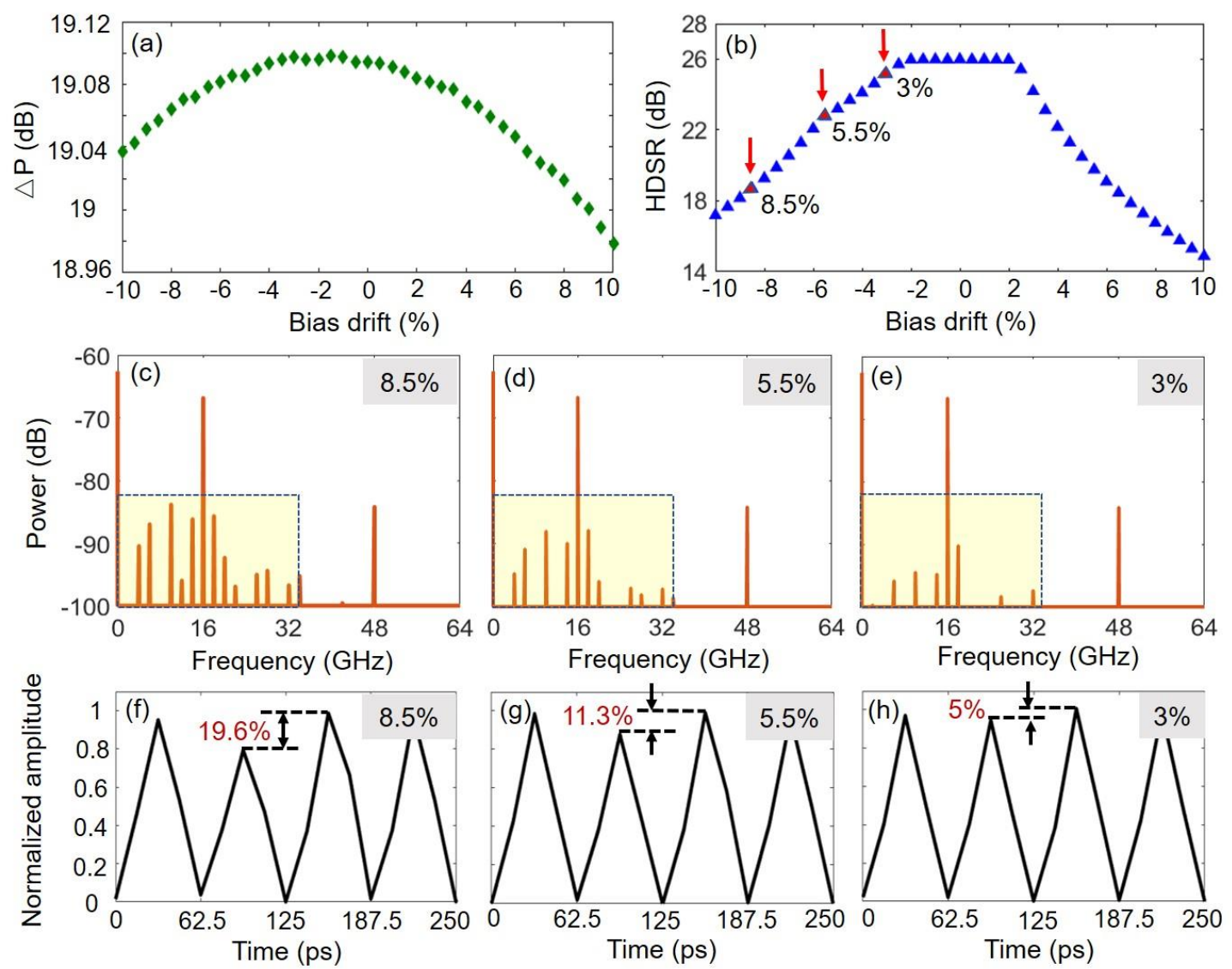

Fig. 7. (a-b) $\triangle P$ and $H D S R$ versus DP-MZM1 bias drift within a range of $\pm 10 \%$. (c-e) Electrical spectra with bias drifts of $8.5 \%, 5.5 \%$, and $3 \%$. (f-h) Corresponding temporal waveforms.

\section{Data Availability}

Data from OptiSystem 10.0 simulations are available upon request from the corresponding author.

\section{DISCUSSION}

This work demonstrates a novel photonic approach to generate a frequency-octupled triangular waveform. The highlight of the generator is the high frequency multiplying, meanwhile the desired modulation indices are unfixed (for DP-MZMs) and small. By using a 2-GH RF driving signal, a 16-GHz triangular waveform is successfully obtained. To analyze the stability and feasibility of the proposed triangular waveform generator, we discussed the phase imbalance of the hybrid coupler and the bias drift of the MZM. It proves that the generator has a relatively large tolerable error range, $\pm 2 \%$ phase imbalance and $\pm 3 \%$ bias drift, respectively. The proposed approach is an efficient and promising candidate for the triangular waveform generator, providing a viable solution for the future ultra-high frequency applications.

\section{REFERENCES}


[1] Rashidinejad, A., Li, Y., and Weiner, A. M. Recent advances in programmable photonic-assisted ultrabroadband radiofrequency arbitrary waveform generation. IEEE $J$. Quantum Electron. 52, 1-17 (2016).

[2] Yao, J. P. Photonic generation of microwave arbitrary waveforms. Opt. Commun. 284, 37233736 (2011).

[3] Bhamber, R. S., Latkin, A. I., Boscolo, S. \& Turitsyn, S. K. All-optical TDM to WDM signal conversion and partial regeneration using XPM with triangular pulses. Optical Communication: The 34th European Conference on Optical Communication (ECOC), Brussels, Belgium. IEEE. (https://doi.org/10.1109/ECOC.2008.4729368) (2008, Sept 21-25).

[4] Latkin, A. I., Boscolo, S., Bhamber, R. S. \& Turitsyn, S. K. Optical frequency conversion, pulse compression and signal copying using triangular pulses. Optical Communication: The 34th European Conference on Optical Communication (ECOC), Brussels, Belgium. IEEE. (https://doi.org/10.1109/ECOC.2008.4729140) (2008, Sept 21-25).

[5] Latkin, A. I., Boscolo, S., Bhamber, R. S. \& Turitsyn, S. K. Doubling of optical signals using triangular pulses. J. Opt. Soc. Am. B. 26, 1492-1496 (2009).

[6] Ye, J. et al. Photonic generation of triangular-shaped pulses based on frequency-to-time conversion. Opt. Lett. 36, 1458-1460 (2011).

[7] Jiang, H. Y. et al. Photonic arbitrary waveform generation based on crossed frequency to time mapping. Opt. Express. 21, 6488-6496 (2013).

[8] Pan, S. et al. Optoelectronic oscillator based on polarization modulation. Fiber Integr. Opt. 34, 185-203 (2015).

[9] Wang, W. Y. et al. Triangular microwave waveforms generation based on an optoelectronic oscillator. IEEE Photon. Technol. Lett. 27, 522-525 (2015).

[10] $\mathrm{Wu}$, T. et al. Simultaneous triangular waveform signal and microwave signal generation based on dual-loop optoelectronic oscillator. IEEE Photon. J. 8, 1-10 (2016).

[11] Zhang, F. et al. Triangular pulse generation by polarization multiplexed optoelectronic oscillator. IEEE Photon. Technol. Lett. 28, 1645-1648 (2016).

[12] Jiang, Y. et al. Photonic microwave waveforms generation based on time-domain processing. Opt. Express. 23, 19442-19452 (2015).

[13] Jiang, Y. et al. Photonic generation of triangular waveform utilizing time-domain synthesis. IEEE Photon. Technol. Lett. 27, 1725-1728 (2015).

[14] Zhang, F. et al. Time-domain waveform synthesis using a dual-polarization modulator. IEEE Photon. Technol. Lett. 23, 2689-2692 (2016).

[15] Jiang, Y. et al. Photonic microwave waveforms generation based on time-domain processing. Optics Express. 23, 19442-19452 (2015).

[16] He, Y. et al. Photonic microwave waveforms generation based on two cascaded single-drive Mach-Zehnder modulators. Opt. Express. 26, 7829-7841 (2018).

[17] Li, J. et al. Performance analysis of a photonic-assisted periodic triangular-shaped pulses generator. J. Lightw. Technol. 30, 1617-1624 (2012).

[18] Zhai, W. et al. Photonic Generation and Transmission of Frequency-Doubled Triangular and Square Waveforms Based on Two Mach-Zehnder Modulators and a Sagnac Loop. J. Lightwave Technol. 37, 1937-1945 (2019).

[19] Gao, Y. et al. Photonic Generation of Triangular Pulses Based on Phase Modulation and Spectrum Manipulation. IEEE Photonics Journal. 8, 1-9 (2016). 
[20] Li, J. et al. Frequency-doubled triangular-shaped waveform generation based on spectrum manipulation. Opt. Lett. 41, 199-202 (2016).

[21] Yuan, J. et al. Photonic Generation of Triangular-shaped Waveform Based on External Modulation. Scientific Reports. 8, 1-10, (2018).

[22] Liu, X. Photonic generation of triangular-shaped microwave pulses using SBS-based optical carrier processing. J. Lightwave Technol. 32, 3797-3802 (2014).

[23] Yuan, J. et al. A photonic-assisted periodic triangular-shaped pulses generator based on FWM effect in an SOA. Opt. Comm. 381, 450-456 (2016).

[24] Liu, J. et al. Photonically assisted microwave waveform generation by gain-transparent SBS-induced carrier processing. Opt. Lett. 42, 3852-3855 (2017).

[25] Yuan, J. et al. Research on photonic generation of quadrupling triangular-shaped waveform using external modulation. Optical Fiber Technology. 45, 352-358 (2018).

[26] Shih, P. T. et al. A continuously tunable and filterless optical millimeter-wave generation via frequency octupling. Optics Express. 17, 19749 (2019).

[27] Meslener, G. et al. Chromatic Dispersion Induced Distortion of Modulated Monochromatic Light Employing Direct Detection, IEEE J. Quant. Electron. 20, 1208-1216 (1984).

\section{Acknowledgements}

This work is jointly supported by the National Natural Science Foundation of China (NSFC) $(61671415,61701448)$.

\section{Author Contributions}

Jin Yuan conceived the idea of the research. Jin Yuan performed all the numerical simulations. Jin Yuan, Jianxun Su and Zengrui Li analyzed the data and wrote the manuscript. Zengrui Li supervised the project. All authors discussed the results and contributed to the manuscript.

\section{Additional Information}

Competing Financial Interests: The authors declare that they have no competing interests. 
Figures
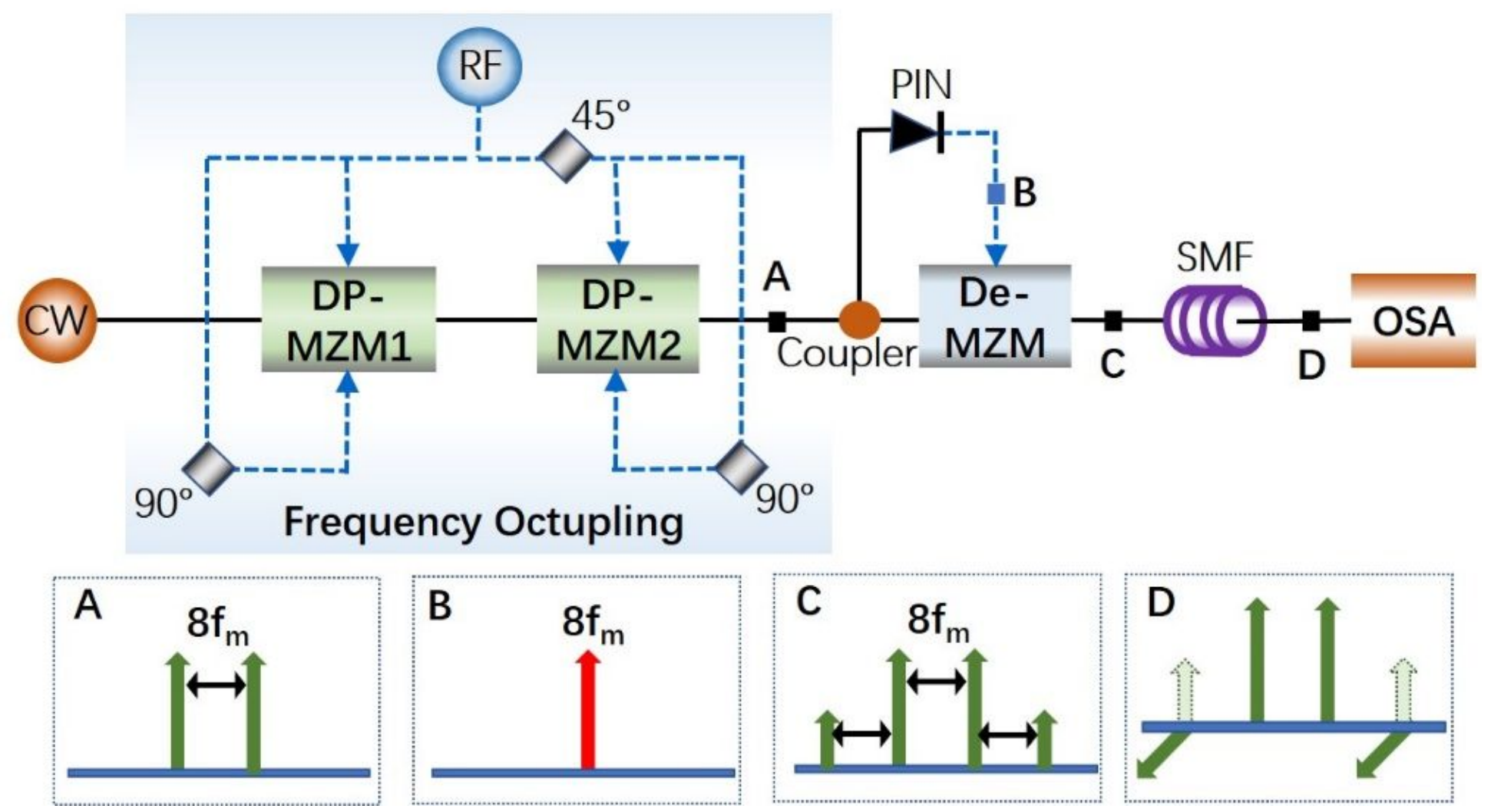

Figure 1

Schematic diagram of the proposed triangular waveform generator.
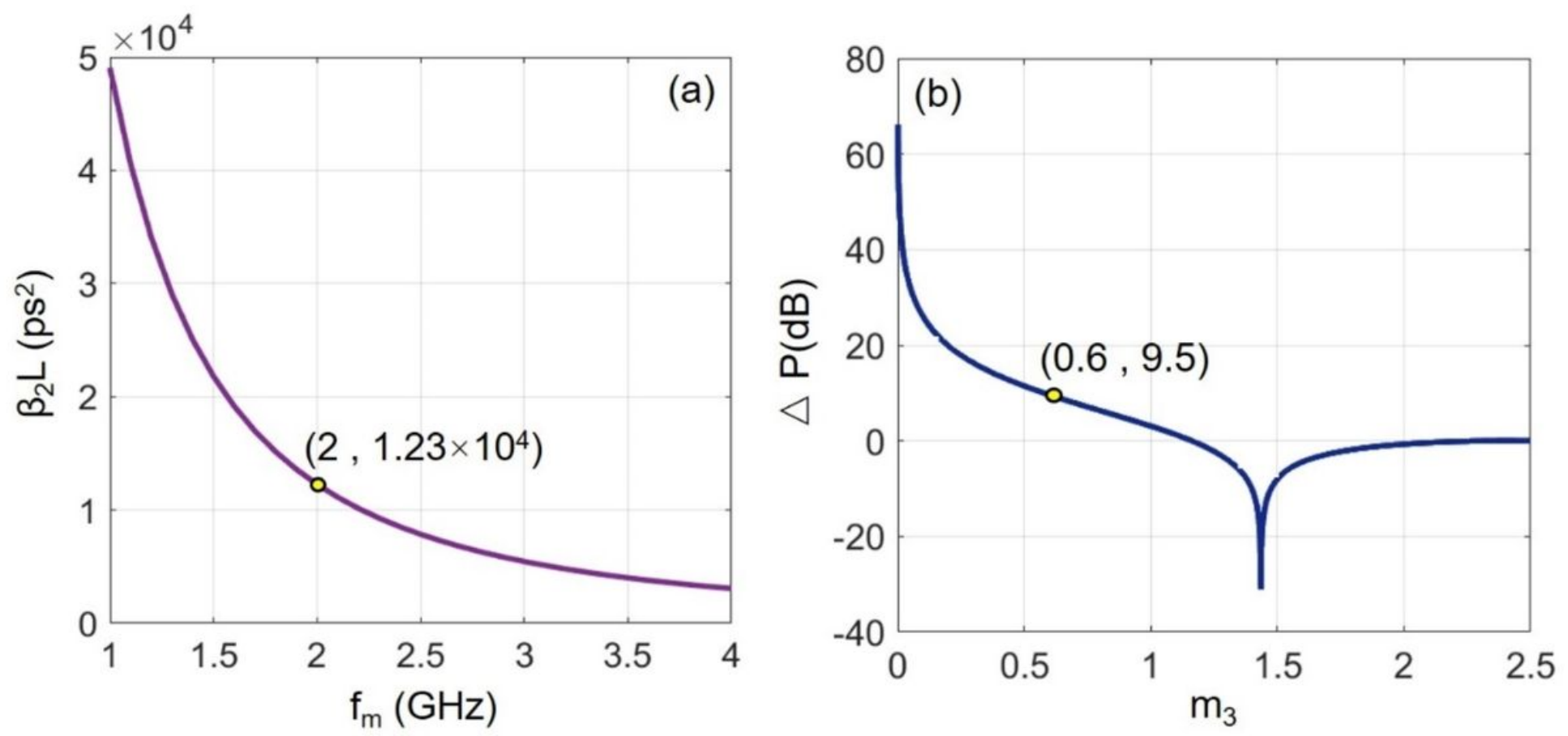
Figure 2

(a) Values of $\beta 2 L$ versus RF driving frequency, $\mathrm{fm}$. (b) Values of $\triangle P(\mathrm{~dB})$ versus modulation index of DeMZM, m3.
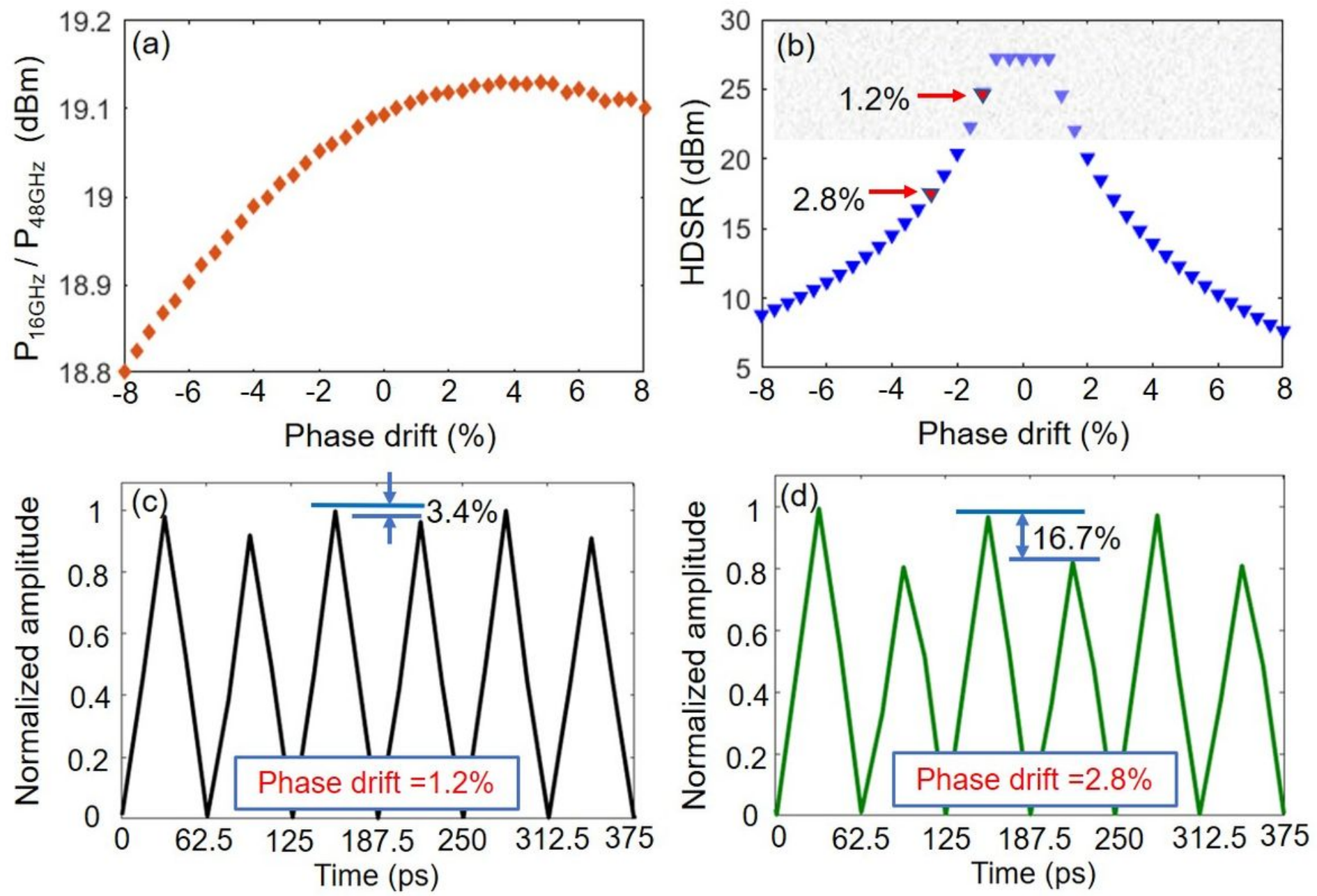

Figure 6

(a-b) $\triangle P$ and HDSR versus phase drift within a range of $\pm 8 \%$. (c-d) Temporal waveforms of the generated signal when phase drifts are $1.2 \%$ and $2.8 \%$. 

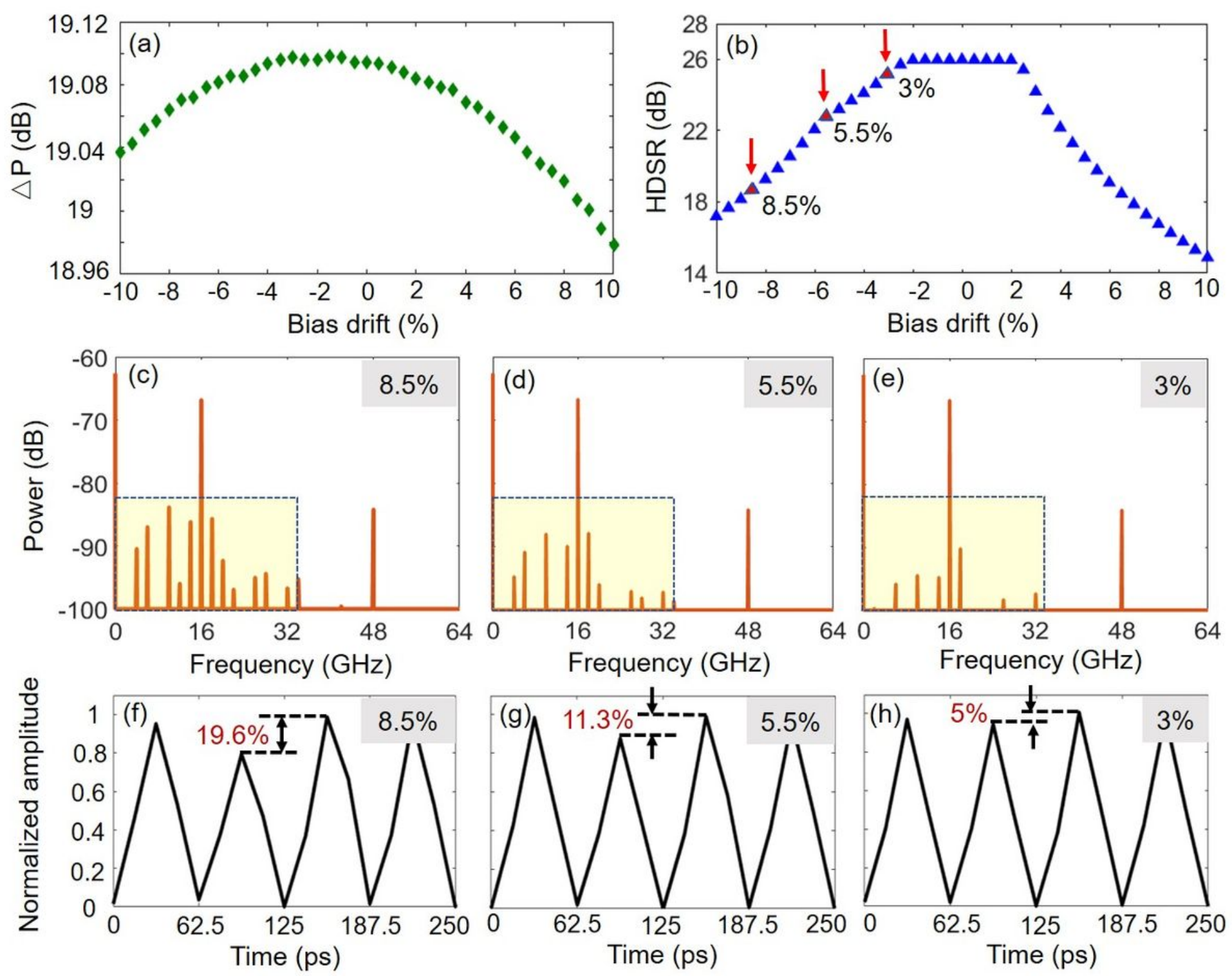

Figure 7

(a-b) $\triangle P$ and HDSR versus DP-MZM1 bias drift within a range of $\pm 10 \%$. (c-e) Electrical spectra with bias drifts of $8.5 \%, 5.5 \%$, and $3 \%$. (f-h) Corresponding temporal waveforms.

\section{Supplementary Files}

This is a list of supplementary files associated with this preprint. Click to download.

- Supplementaryinformation.docx 